\section{The Role of mir-152 and DNMT1 in Gastric Cancer Cell Proliferation and Invasion}

\author{
Zhenkai Wang ${ }^{1,3}$, Yaqin Ye ${ }^{2}$, Shaodong Wang ${ }^{1}$, Hui Shi ${ }^{1}$, Boshi \\ Yuan ${ }^{1}$, Hui Wang ${ }^{1}$, Lin Wu ${ }^{1}$ and Fangyu Wang ${ }^{1^{*}}$ \\ ${ }^{1}$ Nanjing School of Clinical Medicine, Southern Medical University, Nanjing, \\ Jiangsu Province, China
}

${ }^{2}$ Fujian Health Vocational and Technical College, Fuzhou, Fujjian Province, China

${ }^{3}$ Nanjing city hospital of traditional Chinese medicine, Nanjing , Jiangsu Province, China

\begin{abstract}
Background: Multimolecular mechanisms and multifactorial are involved in Gastric Cancer (GC) initiation and progress. Previous studies showed that miR-152 expression was deregulated in many tumors. Similar to dysregulated expression of miRNA, Over expression of DNA methyltransferase member plays crucial roles in GC. In this study we aimed to investigate how miR-152 and DNMTs act on GC
\end{abstract}

Methods: In our study, the expression of miR-152 and DNMT1 were firstly detected in 80 primary GC tissues and their corresponding non-tumor tissues by in situ hybridization, Western blot, immunohistochemistry and quantitative real-time PCR analyzed the difference between the expression and clinicopathological features. Next, the effects of miR-152 on GC cell growth were evaluated by gain function analysis. Then analyzed the expression and relation of miR-152 and DNMT1 in different Gastric cancer cells by quantitative real-time PCR and Western blot. Finally, the target genes of miR-152 in GC cells were explored.

Results: The study demonstrated that miR-152 expression was significantly down regulated in GC tissues compared with the corresponding non tumor tissues. We also showed that miR-152 expression was significantly down regulated in GC cell lines. Ectopic

*Corresponding author: Fangyu Wang, Nanjing School of Clinical Medicine Southern Medical University, Nanjing, China, Tel: +86 25 52155853; Fax: +86 25 52155853; E-mail: wangfy65@nju.edu.cn; wangfangyuand@163.com

Citation: Wang Z, Ye Y, Wang S, Shi H, Yuan B, et al. (2018) The Role of mir-152 and DNMT1 in Gastric Cancer Cell Proliferation and Invasion. J Gastroenterol Hepatology Res 3: 011.

Received: November 04, 2017; Accepted: January 11, 2018; Published: January 31,2018 expression of miR-152 suppressed GC cell proliferation, colony formation and invasion. Moreover, we identified DNA Methyltransferase 1 (DNMT1) as a direct target gene of miR-152 in GC cell. DNMT1 expression was unregulated in GC tissues and cells. Furthermore, the expression level of miR-152 was negatively correlated with the expression level of DNMT1 in GC tissues $\left(R^{2}=0.546, P<0.001\right)$. Moreover, DNMT1 over expression rescued the effect of miR-152 mediated GC cell proliferation, colony formation and invasion.

Conclusion: Our finding demonstrated that miR-152 might play as a tumor suppressor miRNA partly through targeting DNMT1 expression. Restoration of miR-152 may be a potential therapeutic strategy for GC patients in the future.

Keywords: DNMT1; Gastric cancer; Invasion; miR152; Proliferation

\section{Introduction}

Gastric cancer is the fourth most common human malignant disease and the second leading cause of cancer related mortality worldwide on the basis of the most recent statistics, with particularly high incidences and mortality rates in eastern Asia [1-4]. Gastric cancer is a multifactorial disease. Genetic predisposition and environmental factors have been recognized to be involved in gastric cancer initiation and progress. Large scale multidisciplinary investigations on gastric cancer have indicated that gastric cancer is a multiple gene disease and a series of molecules are involved in gastric cancer carcinogenesis $[5,6]$. Nevertheless, relatively little is known about how specific molecules are involved in the initiation and progression of gastric cancer. Consequently, elucidation of the molecular mechanisms of the carcinogenesis of gastric cancer will be benefit for enhancing prevention, detection and treatment of this disease.

MicroRNAs (miRNAs) constitute a dominating class of small RNAs in most somatic tissues. In RNA silencing, miRNA functions play as a guide to induce translational repression, mRNA deadenylation and mRNA decays by base pairing with its target mRNAs [7]. miRNA - binding sites are usually located in the 3 Untranslated Region (UTR) of mRNAs [8]. More than $60 \%$ of human protein-coding genes contain at least one conserved miRNA - binding site, and considering that numerous non-conserved sites also exist, most protein-coding genes may be under the control of miRNAs [9]. A lot of study suggested that miRNA plays crucial roles in many biological processes such as development, inflammation, differentiation, cell proliferation, apoptosis and invasion [10-13]. Thus, their dysregulation is often associated with human diseases, including cancer and neurodevelopmental disorders $[14,15]$.

Emerging evidences have demonstrated that miRNA are deregulated in various cancers including breast cancer, gallbladder cancer, bladder cancer, cutaneous squamous cell carcinoma, laryngeal cancer, rhabdomyosarcoma and GC [16-21]. miRNA can act as a tumor suppressor gene or oncogene in the initiation and development of tumors $[12,22,23]$. Recently, increasing studies have demonstrated that miR-152 are decreased in various tumor types, indicating that they have the potential to act as tumor-suppressor miRNAs [24-26]. miR152 plays an important role in various tumors [27-29]. However, the 
expression and functional role of miR-152 in GC is still unknown. In this study, we studied the relation between miR-152 and DNMT1 in $\mathrm{GC}$ cell proliferation and invasion to show the role of miR-152 and DNMT1 in the development of GC.

\section{Materials and Methods}

\section{Tissue samples}

Eighty primary GC tissues and their corresponding non-tumor tissues were collected from our department between 2012 and 2015. Regarding the AJCC system, these patients were adapted to the pathological TNM (pTNM) staging system. Written informed consent was collected from all patients, and this study was approved by the Ethics Committee of Jin Ling Hospital. The approval number of the ethics committee was JINLING 2016 - 082. These samples were immediately frozen and stored in liquid nitrogen.

\section{In Situ Hybridization (ISH)}

In situ detection of miR-152 was performed on paraffin sections using a DIG-labeled miRCURY ${ }^{\mathrm{TM}}$ Detection probe according to the manufacturer's instructions (Exiqon). Eighty cases of gastric cancer were selected. The sections were deparaffinized and deproteinated, which was followed by prehybridization, hybridization [hybridization temperature $=$ Tm probe $\left.-21^{\circ} \mathrm{C}\right]$, stringency washing and immunological detection. The sections were then exposed to a streptavidin-peroxidase reaction system and developed with 3, 3'-Diaminobenzidine (DAB). Slides were counterstained with hematoxylin and analyzed with a Nikon 80i microscope and Nikon NIS Elements F 2.3 software (Nikon).

\section{Immunohistochemistry}

The Streptavidin - Peroxidase (SP) method was adopted and performed. Primary antibodies against DNMT1 (sc-20701), DNMT3A (sc-20703) and DNMT3B (sc-20704) were purchased from Santa Cruz Biotechnology, Inc., (Santa Cruz, CA, USA). The SP and DAB kits were obtained from Beijing Zhongshan Golden Bridge Biotechnology Co., Ltd. (Beijing, China).

\section{Evaluation of staining:}

DNMTs expression was assessed by scoring the staining intensity and stained proportion of the cell nucleus. Staining intensity was recorded as negative $=0$, light $=1$, moderate $=2$ or strong $=3$. The staining proportion was recorded as $1(\leq 25 \%), 2(\leq 50 \%), 3(\leq 75 \%)$ or $4(>75 \%)$. The two values were multiplied for each slide to produce a terminal score. If the score was higher in cancer cells than in matched control cells, this pair of tissues was marked with a ' + ' (corresponding to cancer cells that expressed elevated DNMT levels). The opposite condition was marked with a '-' (corresponding to cancer cells that expressed reduced DNMT levels). If the scores were equal, the pair was marked with a ' 0 ' (corresponding to similar DNMT expression in cancer and control). Terminal scores of 0-3 were defined as negative expression; 4-12 were defined as positive expression.

\section{Cell lines cultured and transfection}

Human GC cell lines (MKN-45, AGS, MKN-28 and HGC-27) and normal gastric mucosa cells line (GES-1) were purchased from Cell Bank of Chinese Academy of Sciences (Beijing, China). The cell lines were cultured in RPMI 1640 medium (Gibco, USA) containing 10\%
Fetal Bovine Serum (FBS). miR-152 mimic and scramble, DNMT1 vector, and control were synthesized by Ribobio (Guangzhou, China). Cell transfection was performed by using Lipofectamine 2000 kit (Invitrogen, USA) following to the manufacturer's information.

\section{Quantitative real-time RT-PCR}

Total RNAs from tissue specimens and cultured cells were extracted with the TRIzol reagent (TaKaRa, Japan). RT was performed according to the instructions for the Prime script Reverse Transcription Reagent Kit (TaKaRa, Japan). qRT-PCR analysis was processed using SYBR Green PCR Master Mix Kit (TaKaRa, Japan) and was conducted on a 7500 HT analyzer (Applied Biosystems, Foster City, CA, USA) in 96-well PCR plates. The mRNA levels of miR-152 and DNMT1 were evaluated based on the CT recordings and were normalized to housekeeping genes U6 and $\beta$-actin levels, respectively. The primers used in our study were synthesized from Ribobio (Guangzhou, China).

The sequences of RT primers were: miR-152: 5'-GTCGTATCCAGTGCAGGGTCC GAGGTATTCGCACTGGATACGCCCAAGT-3' and U6: 5'-AACGCTTCACGA ATTTGCGT-3'. The sequences of PCR primers used were as follows: miR-152 forward 5'-GAGTGCTCAGTGCATGCAG-3' and reverse 5'-GTGCAGGGTCCG AGGT-3'; DNMT1, forward 5'-GCTACCTGGCTAAAGTCAAA $-3^{\prime}$ and reverse 5'-CCATTCCCACTCTACGG-3'; $\beta$-Actin, forward 5'-AGTGTGCGTGGCATCC GCAAAG-3' and reverse 5'-ATCCACATCTGCTGGAAGGTGGC-3'; U6 forward 5'-GCTTCGGCAGCACATATACTAAAAT-3' and reverse 5'-CGCTTCACGAATT TGCGTGTCAT-3'. The relative miR-152 expression was normalized to U6 SnRNA and the expression of DNMT1 was normalized to $\beta$-Actin.

\section{Construction of Vectors}

The 3' untranslated regions (3'-UTRs) of DNMT1 containing an intact miR-152 recognition sequence were amplified by PCR from genomic DNA and the PCR product was subcloned into a GL3 - promoter vector (Promega Corp., Madison, WI) immediately downstream of the luciferase gene. The primers used were 5'-GCTCTAGATCCCTGCACCTACCG-3' (forward) and 5'-GCTCTAGCATA AAGT TTAATTTCCACTC-3' (reverse). A pGL3 construct containing the DNMT1 3'-UTR with point mutations in the seed sequence was synthesized with a Quik-Change site-directed mutagenesis kit (Stratagene, Agilent Technologies, Palo Alto and CA). The has-miR-152 expression vector pcDNA3.1- has miR-152 contains pri-miR-152 and some of its flanking sequences and the sequences were cloned into a pcDNA3.1 vector (Promega). This vector can simulate the natural state of the stable expression of miRNA. The primers used were 5'-CCCTGCTCGAGGTGGCAC-3' (forward) and 5'-GGGGCTGAAGTTCTGGGTC-3' (reverse). The plasmid Enhanced Green Fluorescent Protein (pEGFP) - HBx vector was constructed in our laboratory previously [26]. The complementary DNA encoding DNMT1 was PCR-amplified with PfuUltra II Fusion HS DNA polymerase (Stratagene) with the primers 5'-GGGGTACCATGCCGGCGCGTACCGC-3' (forward) and 5'-GCGAATTCCT AGTCCTTAGCAGCTTCCTCCTCC-3' (reverse) and was subcloned into the pcDNA3.1 vector. The resulting DNMT1 expression vector was confirmed by sequencing. 


\section{Dual-luciferase reporter assay}

Cells were cultured in the 96-well plate and were transfected with pLuc-3'-UTR, renilla and miR-448 mimic or scramble by using Lipofectamine 2000 kit (Invitrogen, USA) following to the manufacturer's information. After $48 \mathrm{~h}$, luciferase activities were analyzed by using a dual-luciferase reporter analysis system (Promega, USA). The relative luciferase activity was normalized to renilla luciferase activity.

\section{Western blot}

Protein was isolated from cell or tissue using RIPA buffer. Total protein was electrophoresed by $12 \%$ SDS and transferred to PVDF membranes (Millipore, USA). After blocking with 10\% nonfat milk, the membrane was incubated with the primary antibody. The antibody was shown as the following:

DNMT1 and $\beta$-Actin (Abcam, England). Blots were measured by using the ECL system.

\section{Cell growth and colony formation}

Cell proliferation was detected using the Cell Counting Kit - 8 kits (CCK - 8 kits, Dojindo, Japan). The cell was cultured in the 96 - well plate, and cell proliferation was detected on 1, 2, 3, 4, 5, 6 and $7 \mathrm{~d}$. The absorbance was detected at $450 \mathrm{~nm}$. For cell colony formation, cells were seeded in the 6 - well plate and kept in RPMI 1640 medium for 2 weeks. Colonies were stained with crystal violet and counted.

\section{Cell invasion}

Cell invasion was performed using trans well chambers. Cells were plated in the upper of the membranes coated with Matrigel (BD Biosciences, MD). The upper chamber was not supplemented with FBS, and the $10 \%$ FBS was added to the lower membrane. After incubating for $48 \mathrm{~h}$, the cells in the lower membrane were stained with $1 \%$ crystal violet (Sigma, USA) and then counted.

\section{Statistical analysis}

The expression level of miR-152 in cancer relative to its nontumorous control was compared. Results presented are expressed as mean \pm SD (Standard Deviation). Student's t-test was performed. The Wilcoxon signed rank test (for immunohistochemistry analysis), $\mathrm{X}^{2}$ test and Fisher's exact test (for association analysis between DNMT expression and clinical parameters of GC) were used to measure the difference between two groups, and differences between more than two groups were assessed using one-way ANOVA. Correlations between expression levels of miR-152 and DNMT1 in GC and non-tumor tissues were evaluated by Pearson's correlation. $\mathrm{P}<0.05$ was considered significant.

\section{Results}

\section{miR-152 expression was down regulated in the GC cell and tissue}

We firstly analyzed the expression levels of miR-152 in 80 pairs of GC tissue specimens and matched adjacent normal non-tumor tissues by ISH. The expression levels of miR-152 in GC tissues were significantly decreased when compared to the matched normal tissues (Figure 1A).

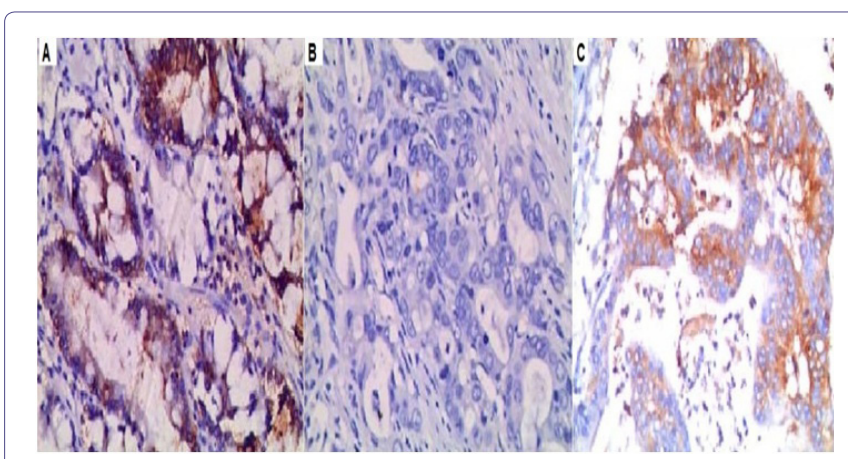

Figure 1A: miR-152 expression was downregulated in the GC cell and tissue.

Detection of miR-152 by in situ hybridization in serial sections from gastric cancer tissue and its matched non-tumor adjacent tissue. Images are overlay images with brown color representing miR-152 expression. Data are presented as mean \pm SD from at least three separate experiments. The miR152 expression in non-tumor tissue (A), Lower expression in GC tissue (B) higher expression in GC tissue (C) compared to non-tumor tissue.

Clinical pathologic features of gastric cancer patient are listed in table 1 .

\begin{tabular}{|c|c|c|c|c|c|}
\hline \multirow{2}{*}{ Parameter } & \multirow{2}{*}{ N (\%) } & \multicolumn{2}{|c|}{ miR-152 } & \multirow{2}{*}{$\mathbf{X}^{2}$} & \multirow{2}{*}{$\mathbf{P}$} \\
\hline & & Lower & Higher & & \\
\hline \multicolumn{6}{|l|}{ Gender } \\
\hline Male & $63(78.75)$ & $53(66.25)$ & $10(12.5)$ & 3.156 & 0.093 \\
\hline Female & $17(21.25)$ & $11(13.75)$ & $6(7.45)$ & & \\
\hline \multicolumn{6}{|l|}{ Age (years) } \\
\hline$\leq 60$ & $28(35)$ & $23(28.75)$ & $5(6.25)$ & 4.515 & 0.034 \\
\hline$>60$ & $52(65)$ & $41(51.25)$ & $11(13.75)$ & & \\
\hline \multicolumn{6}{|l|}{ Tumor location } \\
\hline Proximal & $42(52.5)$ & $33(41.25)$ & $9(11.25)$ & 0.965 & 0.617 \\
\hline Middle & $21(26.25)$ & $16(20)$ & $5(6.25)$ & & \\
\hline Distal third & $17(21.25)$ & $15(18.75)$ & $2(2.5)$ & & \\
\hline \multicolumn{6}{|l|}{ Tumor size (cm) } \\
\hline$\leq 3 \mathrm{~cm}$ & $41(51.25)$ & $29(36.25)$ & $12(15)$ & 3.405 & 0.065 \\
\hline$>3 \mathrm{~cm}$ & $39(48.75)$ & $35(43.75)$ & $4(5)$ & & \\
\hline \multicolumn{6}{|l|}{$\begin{array}{l}\text { Pathological } \\
\text { differentiation }\end{array}$} \\
\hline High & $37(46.25)$ & $31(38.75)$ & $6(7.5)$ & 1.036 & 0.596 \\
\hline Moderate & $36(45)$ & $27(33.75)$ & $9(11.25)$ & & \\
\hline Poor & $7(8.75)$ & $6(7.5)$ & $1(1.25)$ & & \\
\hline \multicolumn{6}{|l|}{ Depth of invasion } \\
\hline Mucosa and muscular & $18(22.5)$ & $10(12.5)$ & $8(10)$ & 8.674 & 0.003 \\
\hline Submucosa and serosa & $62(77.5)$ & $54(67.5)$ & $8(10)$ & & \\
\hline \multicolumn{6}{|l|}{ Lymph node metastasis } \\
\hline No & $30(37.5)$ & $14(17.5)$ & $16(20)$ & 33.333 & 0 \\
\hline Yes & $50(62.5)$ & $50(62.5)$ & 0 & & \\
\hline \multicolumn{6}{|l|}{ TNM stage } \\
\hline $\mathrm{I}+\mathrm{II}$ & $35(43.75)$ & $19(23.75)$ & $16(20)$ & 25.714 & 0 \\
\hline $\mathrm{III}+\mathrm{IV}$ & $45(56.25)$ & $45(56.25)$ & 0 & & \\
\hline
\end{tabular}

Table 1: Correlation between miR-152 expression and clinicopathological features in primary GC patients. 
Lower miR-152 expression levels were detected in $64(80 \%)$ of $80 \mathrm{GC}$ cases, and were significantly correlated with the GC invasive depth and TNM stage. Significant down regulation levels of miR152 was also observed in 50 GC cases with Lymph Node-metastasis (LN) positive patients when compared with $30 \mathrm{LN}$ negative patients. However, we did not observe any obvious links between miR-152 levels and the patients'sex, tumor location and tumor size. To explore the role of miR-152 in GC development, we used qRT-PCR to test miR-152 expression in 80 matched human GC tissues as well as their corresponding noncancerous mucosa tissues. As shown in figure 1B, compared with the peritumoral normal tissues, miR-152 expression is significantly decreased in cancer samples (mean $\pm \mathrm{SD}=13.2621 \pm$ 2.22 and $1.39 \pm 1.02$, respectively).

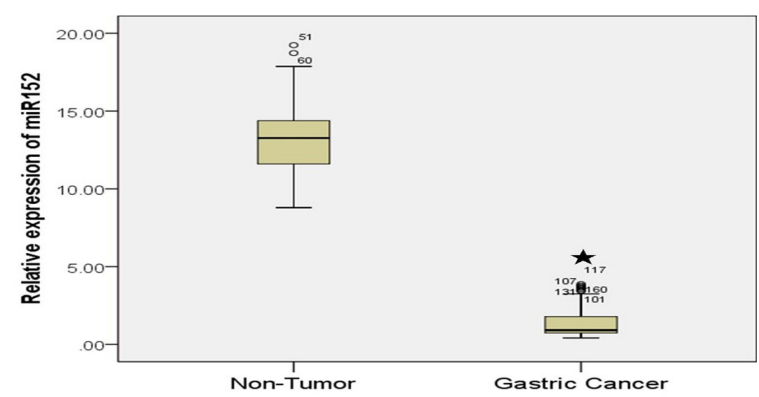

Figure 1b: miR-152 expression was downregulated in the GC cell and tissue.

miR-152 expression was significantly downregulated in GC tissues compared with the paired non-tumor tissues.

Moreover, patients with the early stages (stage I and II) had a much higher miR-152 expression than patients with the advanced stages (stage III and IV) (Figure 1C).

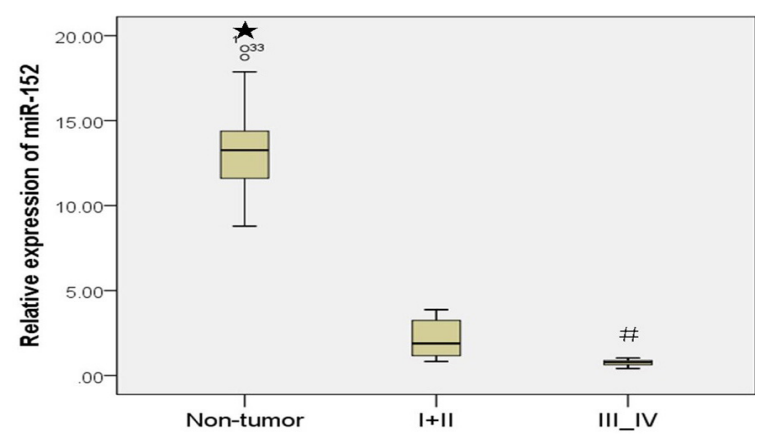

Figure 1c: miR-152 expression was downregulated in the GC cell and tissue.

miR-152 level was significantly lower in patients with advanced stage (stage III and IV) than in patients with early stage (stage I and II). Patients were staged according to the $7^{\text {th }}$ Edition of the AJCC Cancer's TNM Classification.

Unfortunately, due to a lack of follow-up data, the correlation between miR-152 expression and prognosis could not be investigated. In addition, We also demonstrated that miR-152 expression was decreased in the GC cell lines compared with normal gastric mucosa cells line (GES-1) (Figure 1D).

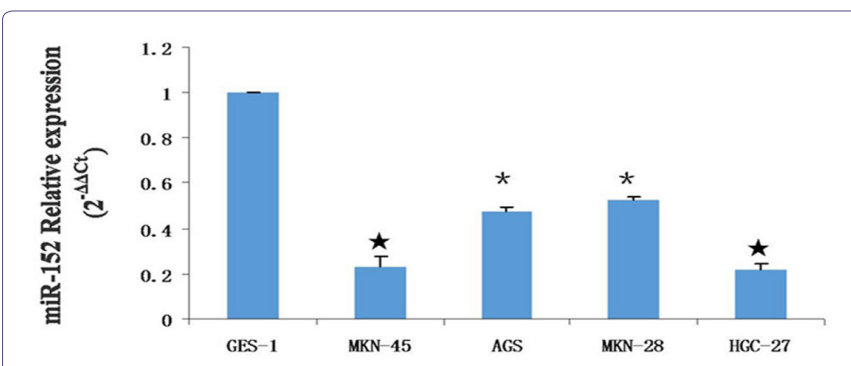

Figure 1D: miR-152 expression was downregulated in the GC cell and tissue.

The expression of miR-152 was downregulated in the GC cell lines (MKN42, AGS, MKN-28 and HGC-27) compared with normal gastric mucosa cells line (GES-1).

*- $\mathrm{P}<0.05, \#-\mathrm{P}<0.01, \star-\mathrm{P}<0.001$.

Among these GC cell lines, MKN-45 and HGC-27 cells displayed the most reduction (approximated $80 \%$ ) in miR-152 expression compared with its expression in GES-1. Hence, further functional analyses on miR-152 were performed in MKN-45 and HGC-27 cells. Taken together, our data showed that miR-152 expression was downregulated in both human GC tissues and GC cell lines, and its downregulation was correlated with clinicopathological features of GC, which indicates that miR-152 may play a vital role in GC progression and development.

\section{Differential expression of DNMT family members in gastric cancer}

GC and matched mucosal tissue expressed DNMT proteins (Figure 2). Notably, DNMT protein distribution was observed primarily in the nucleus of cancerous tissue and in the nucleus and cytoplasm of control tissue.

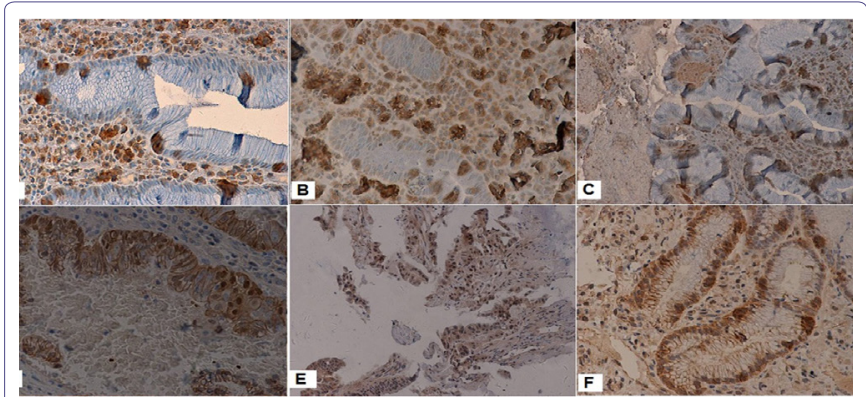

Figure 2: DNA methyltransferase (DNMT1) 1, 3a, 3b expression determined by immunohistochemistry in gastric cancer biopsies.

Gastric carcinomas that express negative DNMT1 (A), DNMT3a (B), DNMT3b (C) and positive DNMT1 (D), DNMT3a (E) and DNMT3b (F) are shown. Original magnification: $\times 200$.

DNMT expression in noncancerous tissue was primarily focused on the proliferating zone of gastric mucosa. DNMTs perform DNA methylation in the nucleus; thus, nuclear staining was investigated. The positive expression rate of DNMT1 was higher than DNMT3a and DNMT3b in GC (Table 2). 
Citation: Wang Z, Ye Y, Wang S, Shi H, Yuan B, et al. (2018) The Role of mir-152 and DNMT1 in Gastric Cancer Cell Proliferation and Invasion. J Gastroenterol Hepatology Res 3: 011

\begin{tabular}{|c|c|c|c|c|}
\hline \multirow{2}{*}{ Parameter } & \multirow{2}{*}{$\mathbf{n}$} & \multirow{2}{*}{$\begin{array}{c}\text { DNMT1 } \\
\text { Expression (\%) }\end{array}$} & \multirow{2}{*}{$\begin{array}{l}\text { DNMT 3a } \\
\text { Expression (\%) }\end{array}$} & \multirow{2}{*}{\begin{tabular}{c|c} 
DNMT 3b \\
Expression (\%) \\
\end{tabular}} \\
\hline & & & & \\
\hline \multicolumn{5}{|l|}{ Gender } \\
\hline Male & 63 & $46(57.5)$ & $23(28.75)$ & $25(31.25)$ \\
\hline Female & 17 & $9(11.25)$ & $8(10)$ & $7(8.75)$ \\
\hline $\mathrm{P}$ & & 0.113 & 0.576 & 0.911 \\
\hline \multicolumn{5}{|l|}{ Age (years) } \\
\hline$\leq 60$ & 28 & $20(25)$ & $12(15)$ & $13(16.25)$ \\
\hline$>60$ & 52 & $35(43.75)$ & $19(23.75)$ & $19(23.75)$ \\
\hline P-value & & 0.704 & 0.635 & 0.389 \\
\hline \multicolumn{5}{|l|}{ Tumor location } \\
\hline Proximal & 42 & $30(37.5)$ & $17(21.25)$ & $18(22.5)$ \\
\hline Middle & 21 & $11(13.75)$ & $8(10)$ & $7(8.75)$ \\
\hline Distal third & 17 & $14(17.5)$ & $6(7.5)$ & $7(8.75)$ \\
\hline $\mathrm{P}$-value & & 1.121 & 0.931 & 0.763 \\
\hline \multicolumn{5}{|l|}{ Tumor size (cm) } \\
\hline$\leq 3 \mathrm{~cm}$ & 41 & $26(32.5)$ & $18(22.5)$ & $16(20)$ \\
\hline$>3 \mathrm{~cm}$ & 39 & $29(36.25)$ & $13(16.25)$ & $16(20)$ \\
\hline P-value & & 0.291 & 0.332 & 0.855 \\
\hline \multicolumn{5}{|l|}{ Differentiation } \\
\hline High & 37 & $29(36.25)$ & $12(15)$ & $15(18.75)$ \\
\hline Moderate & 36 & $22(27.5)$ & $16(20)$ & $14(17.5)$ \\
\hline Poor & 7 & $4(5)$ & $3(3.75)$ & $2(2.5)$ \\
\hline P-value & & 0.222 & 0.559 & 0.809 \\
\hline \multicolumn{5}{|l|}{ Depth of invasion } \\
\hline $\begin{array}{l}\text { Mucosa and } \\
\text { muscular }\end{array}$ & 18 & $8(10)$ & $8(10)$ & $6(7.5)$ \\
\hline $\begin{array}{l}\text { Submucosaand } \\
\text { serosa }\end{array}$ & 62 & $47(58.75)$ & $23(28.75)$ & $26(32.5)$ \\
\hline $\mathrm{P}$-value & & $0.011 *$ & 0.573 & 0.512 \\
\hline \multicolumn{5}{|l|}{$\begin{array}{l}\text { Lymph node } \\
\text { metastasis }\end{array}$} \\
\hline No & 30 & $8(10)$ & $12(15)$ & $14(17.5)$ \\
\hline Yes & 50 & $47(58.75)$ & $19(23.75)$ & $18(22.5)$ \\
\hline P-value & & $0.000^{*}$ & 0.859 & 0.346 \\
\hline \multicolumn{5}{|l|}{ TNM stage } \\
\hline I+II & 35 & $15(18.75)$ & $15(18.75)$ & $17(21.25)$ \\
\hline $\mathrm{III}+\mathrm{IV}$ & 45 & $40(50)$ & $16(20)$ & $15(18.75)$ \\
\hline P-value & & $0.000^{\circ}$ & 0.506 & 0.168 \\
\hline
\end{tabular}

Table 2: Positive DNMT expression and differential clinical characteristics of gastric carcinoma, $\mathrm{n}(\%)$.

According to the TNM classification of malignant tumors, expression of DNMT1 was associated with lymph node metastasis in GC (Table 2). GC samples with features of lymph node metastasis and attributes of late TNM classification were found to express DNMT1 protein at a higher level than other samples. There was no association noted in other DNMTs expression and demographic variables of the carcinoma. So DNMT1 expression was significantly related with depth of invasion, Lymph node metastasis and TNM stage of GC.

\section{DNMT1 expression was upregulated in the GC cell and tissues}

We also showed that the expression level of DNMT1 was increased in the GC cell lines compared with GES-1 (Figures 3A \& 3B).

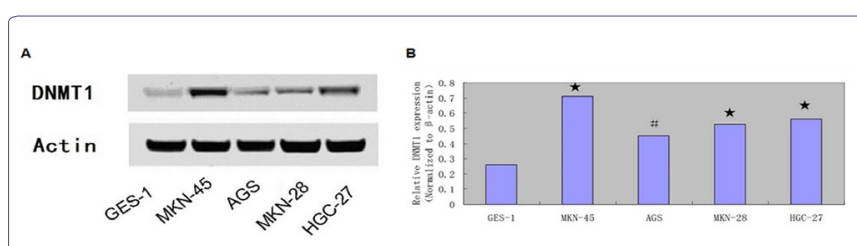

Figure 3: DNMT1 expression was upregulated in the GC cell compared with GES-1.

A) The protein expression of DNMT1 was measured by western blot

(B) The mRNA expression of DNMT1 was measured by qRT-PCR.

$\#-\mathrm{P}<0.05, \star-\mathrm{P}<0.001$.

Moreover, DNMT1 expression was upregulated in 55 cases $(55 / 80 ; 68.75 \%)$ compared with the adjacent tissues (Table 2). There was a negative relationship between the expression level of miR-152 and DNMT1 in the GC tissues $\left(\mathrm{R}^{2}=0.546, \mathrm{P}<0.001\right)$ (Figure 4).

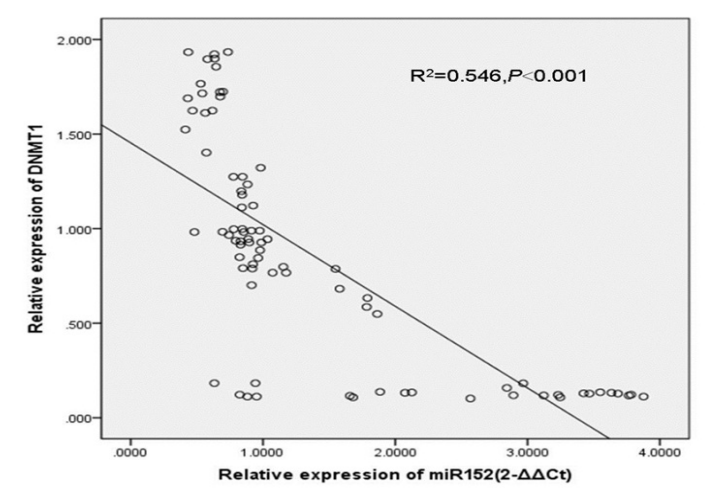

Figure 4: There was negative relationship between the expression level of miR-152 and DNMT1 in the GC tissues.

\section{miR-152 suppressed the GC cell proliferation, colony formation and invasion}

miR-152 expression was upregulated in the GC cell HGC-27 and MKN-45 after being treated with miR-152 mimic and this result confirmed that the efficiency of miR-152 mimic was high (Figure 5A). Over expression of miR-152 suppressed the GC cell HGC-27 and MKN-45 proliferation (Figures 5B \& 5C). Moreover, ectopic expression of miR-152 inhibited the HGC-27 and MKN-45 cell colony formation and invasion (Figures 5D-5G).

\section{DNMT1 was a direct target gene of miR-152 in GC cell}

Bioinformatics assay with Target Scan algorithms predicted DNMT1 as a hypothetical target gene of miR-152 (Figure 6A). In MKN-45 and HGC-27 cells co-transfected with miR-152 mimic or mimic NC and the reporter plasmid, the relative luciferase activity of this reporter with WT DNMT1 3'-UTR was inhibited by miR-152 
mimic; however, this luciferase activity of MT reporter was unaffected (Figures 6B \& 6C). Overexpression of miR-152 suppressed the DNMT1 protein expression in the MKN-45 and HGC-27 cell (Figure 6D).

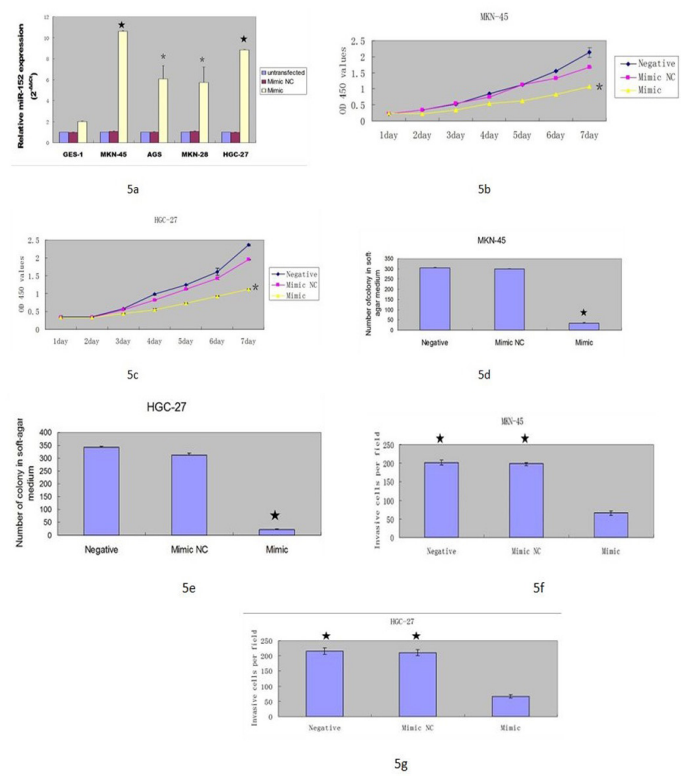

Figure 5: miR-152 suppressed the GC cell proliferation, colony formation and invasion.

A) The expression of miR-152 expression was upregulated in the HGC-27 cell and MKN-45 cell after transfected with miR-152 mimic using qRTPCR.

(B \& C) Overexpression of miR-152 suppressed the MKN-45 and HGC-27 cell proliferation.

(D \& E) Ectopic expression of miR-152 inhibited the MKN-45 and HGC27 cell colony information.

(F \& G) miR-152 overexpression suppressed the MKN-45 and HGC-27 cell invasion.

$$
\star \text { - } \mathrm{P}<0.001, * \text { - } \mathrm{P}<0.05 \text {. }
$$
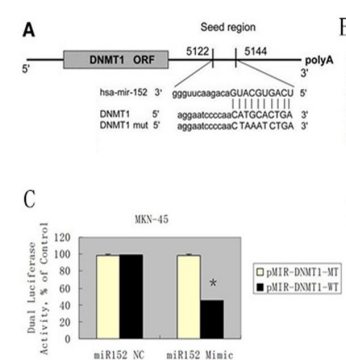

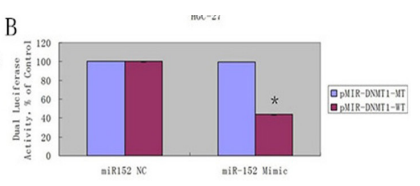

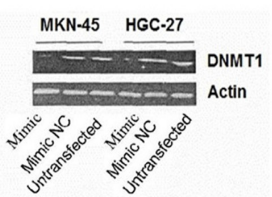

Figure 6: DNMT1 was a direct target gene of miR-152 in GC cell.

A) Bioinformatics assay with Target Scan algorithms predicted DNMT1as a hypothetical target gene of miR-152.

(B \& C) Luciferase activity assays of luciferase vectors with wild-type or mutant DNMT1 3'-UTR were performed after co-transfection with miR152 mimic or mimic NC in the MKN-45 and HGC-27 cell.

(D) Overexpression of miR-152 suppressed the DNMT1 protein expression in the MKN-45 and HGC-27 cell.

* - P $<0.05$ miR-152 mimic and wild-type DNMT1 3'-UTR co-transfection group vs. other three groups.

\section{DNMT1 rescued the effect of miR-152-mediated GC cell proliferation, colony formation and invasion}

DNMT1 expression was upregulated in the pCDNADNMT1- WT 3'-UTR or pCDNADNMT1- MT 3'-UTR treated GES cell (Figures $7 \mathrm{~A} \& 7 \mathrm{~B})$.

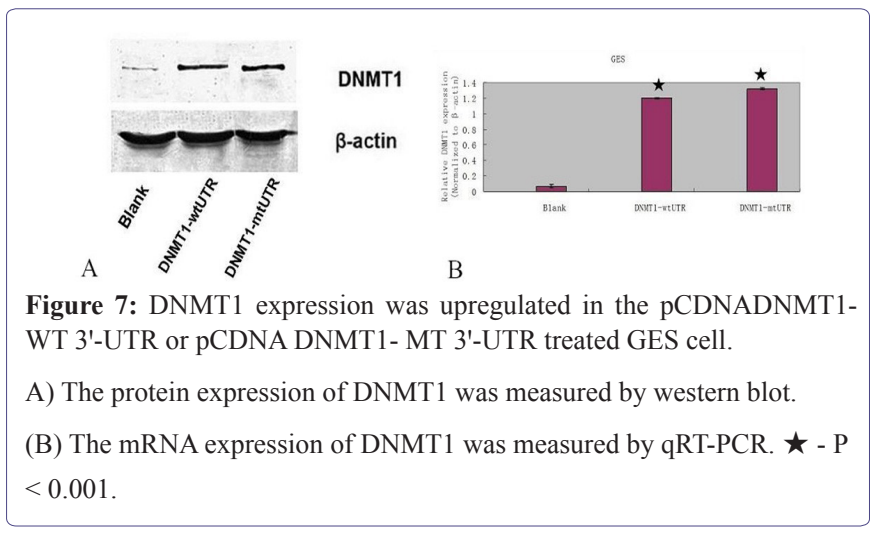

We rescued DNMT1 expression in the miR-152 overexpressing MKN-45 and HGC-27 cell in the pCDNADNMT1- MT 3'-UTR treated group (Figures 8A \& 8B). Furthermore, overexpression of miR152 suppressed the DNMT1 protein expression in the MKN-45 and HGC-27 cell with wt DNMT1 3'-UTR. CCK8 analysis demonstrated that DNMT1 overexpression promoted the miR-152-overexpressing MKN-45 and HGC-27 cell proliferation (Figures 8C \& 8D). Colony formation analysis showed that DNMT1 overexpression increased the miR-152 overexpressing MKN-45 and HGC-27 cell colony formation (Figures $8 \mathrm{E} \& 8 \mathrm{~F}$ ). Invasion analysis demonstrated that ectopic expression of DNMT1 promoted the miR-152-overexpressing MKN45 and HGC-27 cell invasion (Figures 8G \& 8H).

\section{Discussion}

There has been a rapid increase in the number of publications focusing on miR-152 in recent years, which revealed that the expression of miR-152 was inhibited in a variety of tumors, including ovarian cancer [25], endometrial cancer [26], liver cancer [29], non-small lung cancer [28], and colorectal cancer [27]. Here, we investigate the functions of miR-152 in GC and found that miR-152 expression was signifycantly downregulated in GC tissues and cell lines. On the other hand, overexpression of miR-152 significantly inhibited cell proliferation, colony formation and tumor angiogenesis in vitro studies. Su et al., demonstrated that restoration of miR-152 significantly reduced cell proliferation, colony formation, migration and invasion partially via targeting ADAM17 [30]. In addition, miR-152 is also able to target Wnt1 and MMP3 to inhibit cell proliferation in liver cancer cells, thus reducing glioma cell invasion and angiogenesis, respectively [31]. Our study also showed ectopic expression of miR-152 suppressed GC cell proliferation, colony formation and invasion. These findings suggest that miR-152 may potentially function as a tumor suppressor in human cancer. Aberrant DNA hypermethylation of tumor suppressor genes, Global DNA hypermethylation (GDM) and disruption of histone modification patterns are the three most important epigenetic changes contributing to the malignant phenotype [32]. In particular, DNA hypermethylation may be important in the initiation of multiple types 
of cancer [32,33]. Nakajima et al., also found that DNA methylation levels in the gastric mucosae correlate with gastric cancer risk levels [34]. Methylation of mammalian genomic DNA is catalyzed by DNMTs. The mammalian DNMT family includes three active members: DNMT1, DNMT3a and DNMT3b [35,36]. The human DNMT 1, 3a, and $3 \mathrm{~b}$ coordinate mRNA expression in normal tissues and overexpression in tumors [37]. The expression levels of these DNMTs are reportedly elevated in human cancers [36-39]. There is considerable evidence indicating an up-regulation of DNMT1 in cancer [40,41]. In our study, the positive expression rate of DNMT1 was higher than DNMT3a and DNMT3b in GC. And DNMT1 expression was significantly related with depth of invasion, Lymph node metastasis and TNM stage of GC.
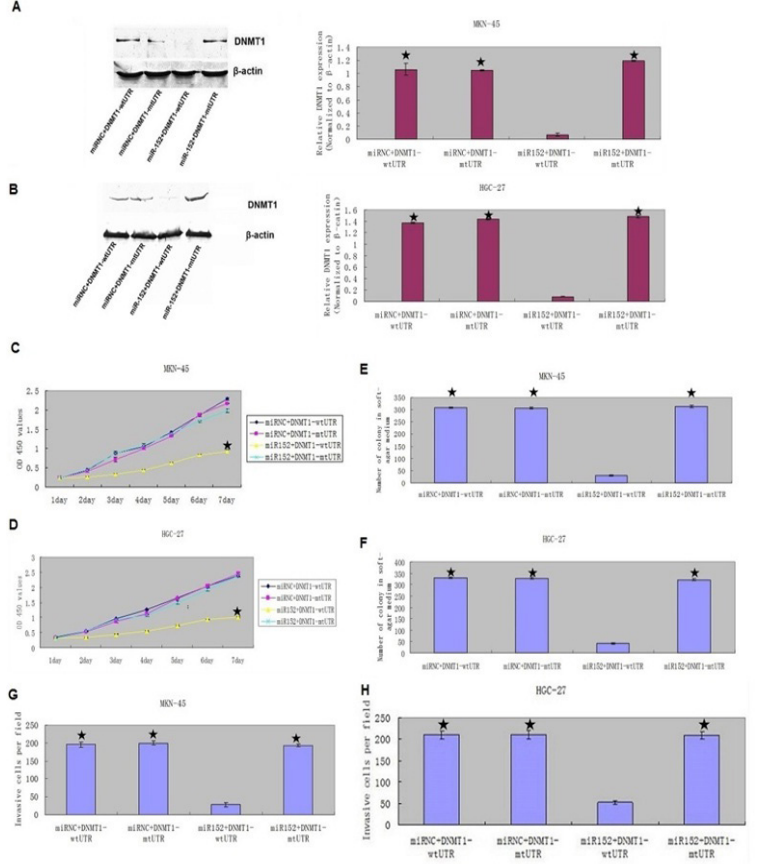

Figure 8: DNMT1 rescued the effect of miR-152-mediated GC cell proliferation, colony formation and invasion.

(A \& B) The expression of DNMT1 was decreased in the MKN-45 and HGC-27 cell after co-transfected with miR-152 mimic and pCDNADNMT1-wt3'-UTR,but which was upregulated after co-transfected with miR152 mimic and pCDNADNMT1-mt3'-UTR, or miR-152 NC and pCDNADNMT1-wt3'-UTR,or miR-152 NC and pCDNADNMT1-mt3'-UTR.

(C \& D) Overexpression of DNMTI promoted the miR-152 overexpressing MKN-45 and HGC-27 cell proliferation compared with the group of decreased expression of DNMT1

(E \& F) DNMT1 overexpression increased the miR-152 overexpressing MKN-45 and HGC-27 cell colony formation compared with the group of decreased expression of DNMT1.

$(\mathrm{G} \& \mathrm{H})$ Ectopic expression of DNMT1 promoted the miR-152-overexpressing MKN-45 and HGC-27 cell invasion compared with the group of decreased expression of DNMT1.

$\star-\mathrm{P}<0.001$, co-transfected with miR-152 mimic and pCDNADNMT1-wt3'-UTR vs. Other three groups.

Recent studies have found that DNMT1 plays important roles in the GC development [42-44]. But there was no evidence to show the relation between DNA hypermethylation and miR-152 in GC. Here, we identified DNMT1 as a direct target gene of miR-152 in GC cell and DNMT1 expression was upregulated in GC tissues and cells. Furthermore, the expression level of miR-152 was negatively correlated with the expression level of DNMT1 in the GC tissues. DNMT1 overexpression rescued the effect of miR-152 mediated GC cell proliferation, colony formation and invasion. These observations are consistent with previous findings that miRNA-152 induces aberrant DNA methylation in hepatitis B virus - related hepatocellular carcinoma and endometrial cancer by targeting DNMT1 [24]. Our findings indicate that miR-152 directly interacted with the DNMT1 and the lower levels of miR-152 expression in GC cells may be one of the reasons for the abnormal expression of DNMT1, which leads to aberrant DNA methylation, contributing to GC tumorigenicity and development.

This study was limited by its small number of patients and cancer-related genes. Thus, larger multi-gene studies are needed to validate our results. Further work is necessary to elucidate the exact function and interaction with other factors to develop strategies for early diagnosis, prevention and treatment of GC. On the other hand, some researches provided evidences that the down expression of miR-152 in NiS-transformed cells was regulated by DNMT1 via promoter hypermethylation, and miR-152 was linked to DNA methylation by directly targeting DNMT1 3'-UTR, revealing a crucial functional crosstalk between miR-152 and the DNMT1 via a double-negative feedback loop is involved in NiS-induced malignant transformation $[45,46]$. miR-152 is located at $17 \mathrm{q} 21.32$, and a typical $\mathrm{CpG}$ is land is around miR-152 promoter. Recently, relatively low expression of miR-152 was shown in gastrointestinal cancer and cholangiocarcinoma $[47,48]$. DNA hypermethylation and downregulation of miR-152 were frequently observed in endometrial cancer cell lines and primary tumors [26]. Aberrant DNA methylation of miR-152 CpG islands was reported to correlate with a poor clinical outcome in Mixed Lineage Leukemia (MLL) - rearranged acute lymphoblastic leukemia [49]. Therefore, the next study needs to identify the influence of DNMT1 on the expression of miR-152 in GC.

In conclusion, miR-152 maybe acts as a tumor suppressor miRNA in the development of GC through inhibiting DNMT1 expression. Therefore, restoration of miR-152 may be a potential therapeutic strategy for GC patients in the future.

Ethical approval The study protocol was approved by the Ethics Committee of Jinlin Hospital (Nanjing, China).

\section{Author Contributions}

Wang ZK wrote the paper; Ye YQ and Wang SD designed and analyzed; Shi $\mathrm{H}$ and $\mathrm{Wu}$ L collected the specimen; Yuan BS and Wang $\mathrm{H}$ reviewed histopathology of specimen.

\section{References}

1. Liang J, Liu X, Xue H, Qiu B, Wei B, et al. (2015) MicroRNA-103a inhibits gastric cancer cell proliferation, migration and invasion by targeting c-Myb. Cell Prolif 48: 78-85.

2. Duan JH, Fang L (2014) MicroRNA-92 promotes gastric cancer cell proliferation and invasion through targeting FXR. Tumour Biol 35: 1101311019 .

3. Liu G, Jiang C, Li D, Wang R, Wang W (2014) MiRNA-34a inhibits EGFR-signaling-dependent MMP7 activation in gastric cancer. Tumour Biol 35: 9801-9806. 
Citation: Wang Z, Ye Y, Wang S, Shi H, Yuan B, et al. (2018) The Role of mir-152 and DNMT1 in Gastric Cancer Cell Proliferation and Invasion. J Gastroenterol Hepatology Res 3: 011

4. Wan X, Ding X, Chen S, Song H, Jiang H, et al. (2015) The functional sites of miRNAs and lncRNAs in gastric carcinogenesis. Tumour Biol 36: 521-532.

5. Tamura G (2006) Alterations of tumor suppressor and tumor-related genes in the development and progression of gastric cancer. World J Gastroenterol 12: 192-198.

6. Wu WK, Cho CH, Lee CW, Fan D, Wu K, et al. (2010) Dysregulation of cellular signaling in gastric cancer. Cancer Lett 295: 144-153.

7. Huntzinger E, Izaurralde E (2011) Gene silencing by microRNAs: contributions of translational repression and mRNA decay. Nat Rev Genet 12: $99-110$.

8. Bartel DP (2009) MicroRNAs: target recognition and regulatory functions Cell 136: 215-233.

9. Friedman RC, Farh KK, Burge CB, Bartel DP (2009) Most mammalian mRNAs are conserved targets of microRNAs. Genome Res 19: 92-105.

10. Yu X, Li Z, Chen G, Wu WK (2015) MicroRNA-10b induces vascular muscle cell proliferation through Akt pathway by targeting TIP30. Curr Vasc Pharmacol 13: 679-686.

11. Song JH , Meltzer SJ (2012) MicroRNAs in pathogenesis, diagnosis, and treatment of gastroesophageal cancers. Gastroenterology 143: 35-47.

12. Deng H, Guo Y, Song H, Xiao B, Sun W, et al., (2013) MicroRNA-195 and microRNA-378 mediate tumor growth suppression by epigenetical regulation in gastric cancer. Gene 518: 351-359.

13. Bin Z, Dedong H, Xiangjie F, Hongwei X, Qinghui Y (2015) The microRNA-367 inhibits the invasion and metastasis of gastric cancer by directly repressing Rab23. Genet Test Mol Biomarkers 19: 69-74.

14. Lujambio A, Lowe SW (2012) The microcosmos of cancer. 482: 347-355.

15. Im HI, Kenny PJ (2012) MicroRNAs in neuronal function and dysfunction. Trends Neurosci 35: 325-334.

16. Li Z, Yu X, Shen J, Law PT, Chan MT, et al. (2015) MicroRNA expression and its implications for diagnosis and therapy of gallbladder cancer. Oncotarget 6: 13914-13921.

17. Yu X, Li Z (2015) The role of microRNAs expression in laryngeal cancer. Oncotarget 6: 232297-232305.

18. Li Z, Yu X, Shen J, Liu Y, Chan MT, et al. (2015) MicroRNA dysregulation in rhabdomyosarcoma: a new player enters the game. Cell Prolif 48 511-516.

19. Itesako T, Seki N, Yoshino H, Chiyomaru T, Yamasaki T, et al. (2014) The microRNA expression signature of bladder cancer by deep sequencing: the functional significance of the miR-195/497 cluster. PloS One 9: 84311.

20. Vrba L, Munoz-Rodriguez JL, Stampfer MR, Futscher BW (2013) miRNA gene promoters are frequent targets of aberrant DNA methylation in human breast cancer. PloS One 8: 54398.

21. Xu X, Wang W, Su N, Zhu X, Yao J, et al. (2015) miR-374a promotes cell proliferation, migration and invasion by targeting SRCIN1 in gastric cancer. FEBS Lett 589: 407-413.

22. Kong WQ, Bai R, Liu T, Cai CL, Liu M, et al. (2012) MicroRNA-182 targets camp-responsive element-binding protein 1 and suppresses cell growth in human gastric adenocarcinoma. FEBS J 279: 1252-1260.

23. Han TS, Hur K, Xu G, Choi B, Okugawa Y, et al. (2015) MicroRNA-29c mediates initiation of gastric carcinogenesis by directly targeting ITGB1. Gut 64: 203-214.

24. Huang J, Wang Y, Guo Y, Sun S (2010) Down-regulated microRNA-152 induces aberrant DNA methylation in hepatitis B virus-related hepatocellular carcinoma by targeting DNA methyltransferase 1. Hepatology 52 $60-70$.
25. Zhou X, Zhao F, Wang ZN, Song YX, Chang H, et al. (2012) Altered expression of miR-152 and miR-148a in ovarian cancer is related to cell proliferation. Oncol Rep 27: 447-454.

26. Tsuruta T, Kozaki K, Uesugi A, Furuta M, Hirasawa A, et al. (2011) miR152 is a tumor suppressor microRNA that is silenced by DNA hypermethylation in endometrial cancer. Cancer Res 71: 6450-6462.

27. Li B, Xie Z, Li B (2016) miR-152 functions as a tumor suppressor in colorectal cancer by targeting PIK3R3. Tumour Biol 37: 10075-10084.

28. Zhang YJ, Liu XC, Du J, Zhang YJ (2015) MiR-152 regulates metastases of non-small cell lung cancer cells by targeting neuropilin-1. Int J Clin Exp Pathol 8: 14235-14240.

29. Dang YW, Zeng J, He RQ, Rong MH, Luo DZ, et al. (2014) Effects of miR-152 on cell growth inhibition, motility suppression and apoptosis induction in hepatocellular carcinoma cells. Asian Pac J Cancer Prev 15: 4969-4976.

30. Su Y, Wang Y, Zhou H, Lei L, Xu L (2014) MicroRNA-152 target ADAM17 to suppress NSCLC progression. FEBS Lett 588: 1983-1988.

31. Zheng X, Chopp M, Lu Y, Buller B, Jiang F (2010) miR-15b and miR152 reduce glioma cell invasion and angiogenesis via NRP-2 and MMP-3. Cancer Lett 329: 146-154.

32. Lujambio A, Esteller M (2007) CpG island hypermethylation of tumor suppressor microRNAs in human cancer. Cell Cycle 6: 1455-1459.

33. Plass C (2002) Cancer epigenomics. Hum Mol Genet 11: 2479-2488.

34. Nakajima T, Maekita T, Oda I, Gotoda T, Yamamoto S, et al. (2006) Higher Methylation Levels in Gastric Mucosae Significantly Correlate with Higher Risk of Gastric Cancers. Cancer Epidemiol Biomarkers Prev 15 2317-2321.

35. Ren J, Singh BN, Huang Q, Li Z, Gao Y, et al. (2011) DNA hypermethylation as a chemotherapy target. Cell Signal 23: 1082-1093.

36. Jin B, Li Y, Robertson KD (2011) DNA methylation: superior or subordinate in the epigenetic hierarchy? Genes Cancer 2: 607-617.

37. Robertson KD, Uzvolgyi E, Liang G, Talmadge C, Sumegi J, et al. (1999) The human DNA methyltransferases (DNMTs) 1, 3a and 3b: coordinate mRNA expression in normal tissues and over expression in tumors. Nucleic Acids Res 27: 2291-2298.

38. el-Deiry WS, Nelkin BD, Celano P, Yen RW, Falco JP, et al. (1991) High expression of the DNA methyltransferase gene characterizes human neoplastic cells and progression stages of colon cancer. Proc Natl Acad Sci USA 88 : 3470-3474.

39. Patra SK, Patra A, Zhao H, Dahiya R (2002) DNA methyltransferase and demethylase in human prostate cancer. Mol Carcinog 33: 163-171.

40. Belinsky SA, Nikula KJ, Baylin SB, Issa JP (1996) Increased cytosine DNA- methyltransferase activity is target-cell-specific and an early event in lung cancer. Proc Natl Acad Sci USA 93: 4045-4050.

41. Baylin SB, Herman JG, Graff JR, Vertino PM, Issa JP (1998) Alteration in DNA methylation: a fundamental aspect of neoplasia. Adv Cancer Res 72: 141-196.

42. He M, Fan J, Jiang R, Tang WX, Wang ZW (2013) Expression of DNMTs and genomic DNA methylation in gastric signet ring cell carcinoma. Mol Med Rep 8: 942-948.

43. Ding WJ, Fang JY, Chen XY, Peng YS (2008) The expression and clinica significance of DNA methyltransferase proteins in human gastric cancer Dig Dis Sci 53: 2083-2089.

44. Mutze K, Langer R, Schumacher F, Becker K, Ott K et al. (2011) DNA methyltransferase 1 as a predictive biomarker and potential therapeutic target for chemotherapy in gastric cancer. Eur J Cancer 47: 1817-1825. 
Citation: Wang Z, Ye Y, Wang S, Shi H, Yuan B, et al. (2018) The Role of mir-152 and DNMT1 in Gastric Cancer Cell Proliferation and Invasion. J Gastroenterol Hepatology Res 3: 011

- Page 9 of 9 •

45. Jaenisch R, Bird A (2003) Epigenetic regulation of gene expression: how the genome integrates intrinsic and environmental signals. Nat Genet 33: 245-254.

46. Ji W, Yang L, Yuan J, Yang L, Zhang M, et al. (2013) MicroRNA-152 targets DNA methyltransferase 1 in NiS-transformed cells via a feedback mechanism. Carcinogenesis 34: 446-453.

47. Chen Y, Song Y, Wang Z, Yue Z, Xu H, et al. (2010) Altered expression of MiR-148a and MiR-152 in gastrointestinal cancers and its clinical significance. J Gastrointest Surg 14: 1170-1179.
48. Braconi C, Huang N, Patel T (2010) MicroRNA-dependent regulation of DNA methyltransferase-1 and tumor suppressor gene expression by interleukin-6 in human malignant cholangiocytes. Hepatology 51: 881-890.

49. Stumpel DJ, Schotte D, Lange-Turenhout EA, Schneider P, Seslija L, et al. (2011) Hypermethylation of specific microRNA genes in MLL-rearranged infant acute lymphoblastic leukemia: major matters at a micro scale. Leukemia 25: 429-439. 